\title{
Metric-affine $f(R)$-gravity with torsion: an overview
}

\author{
Salvatore Capozziello ${ }^{1 *}$ and Stefano Vignolo ${ }^{2}$ \\ ${ }^{1}$ Dipartimento di Scienze Fisiche Università "Federico II" di Napoli and INFN Sez. di Napoli Compl. \\ Univ. Monte S. Angelo Ed. N, via Cinthia I- 80126 Napoli (Italy) \\ 2 DIPTEM Sez. Metodi e Modelli Matematici, Università di Genova Piazzale Kennedy, Pad. D - 16129 \\ Genova (Italy)
}

Received XXXX, revised XXXX, accepted XXXX

Published online XXXX

Key words Extended gravity, torsion, Palatini formalism, initial value problem.

PACS 04.50.+h, 04.20.Ex, 04.20.Cv, 98.80.Jr

\begin{abstract}
Torsion and curvature could play a fundamental role in explaining cosmological dynamics. $f(R)$-gravity with torsion is an approach aimed to encompass in a comprehensive scheme all the Dark Side of the Universe (Dark Energy and Dark Matter). We discuss the field equations in empty space and in presence of perfect fluid matter taking into account the analogy with the metric-affine formalism. The result is that the extra curvature and torsion degrees of freedom can be dealt under the standard of an effective scalar field of fully geometric origin. The initial value problem for such theories is also discussed.
\end{abstract}

Copyright line will be provided by the publisher

\section{Introduction}

The issue to enlarge or revise the General Relativity (GR) comes out since several questions in modern physics could strictly depend on the fact that it is a classical theory. This means that quantum effects (ultra-violet scales) are not considered in GR. Besides, it seems to fail at galactic and cosmological scales (infra-red scales) leading to the disturbing puzzles of Dark Energy and Dark Matter. Quantum effects should be considered in any theory which deals with gravity at a fundamental level. Assuming a $\mathbf{U}_{4}$ manifold, instead of $\mathbf{V}_{4}$, is the first straightforward generalization which tries to include the spin fields into the geometrical framework of GR. The Einstein-Cartan-Sciama-Kibble (ECSK) theory is one of the most serious attempts in this direction [1]. However, this mere inclusion of spin matter fields does not exhaust the role of torsion which gives important contributions in any fundamental theory. For example, a torsion field appears in (super)string theory, if we consider the fundamental string modes; we need, at least, a scalar mode and two tensor modes: a symmetric and an antisymmetric one. The latter one, in the low energy limit, is a torsion field [2]. Furthermore, several attempts of unification between gravity and electromagnetism have to take into account torsion in four and in higher-dimensional theories such as Kaluza-Klein ones [3]. Any theory of gravity considering twistors needs the inclusion of torsion [4] while Supergravity is the natural arena in which torsion, curvature and matter fields are treated under the same standard [5].

Besides, several people agree with the line of thinking that curvature and torsion could play specific roles in cosmological dynamics, at early and late epochs. In fact, the interplay of curvature and torsion naturally gives repulsive contributions to the energy-momentum tensor so that cosmological models become singularity-free and accelerating [6].

All these arguments do not allow to neglect torsion in any comprehensive theory of gravity which takes into account the non-gravitational counterpart of the fundamental interactions. However, in most papers, a clear distinction is not made among the different kinds of torsion. Usually torsion is simply related to the

\footnotetext{
* Corresponding author E-mail: capozziello@na.infn.it
} 
spin-density of matter, but, very often, it assumes more general meanings. It can be shown that there are many independent torsion tensors with different properties [8].

On the other hand, the issue to extended GR to more general actions with respect to the Hilbert-Einstein one is revealing a very fruitful approach. From a conceptual point of view, there is no a priori reason to restrict the gravitational Lagrangian to a linear function of the Ricci scalar $R$, minimally coupled with matter. The idea that there are no "exact" laws of physics but that the Lagrangians of physical interactions are "stochastic" functions - with the property that local gauge invariances (i.e. conservation laws) are well approximated in the low energy limit and that physical constants can vary - has been taken into serious consideration [9].

Beside fundamental physics motivations, all these theories have acquired a huge interest in cosmology due to the fact that they "naturally" exhibit inflationary behaviors able to overcome the shortcomings of Cosmological Standard Model [10]. Furthermore, dark energy models mainly rely on the implicit assumption that Einstein's GR is the correct theory of gravity, indeed. Nevertheless, its validity at the larger astrophysical and cosmological scales has never been tested [11], and it is therefore conceivable that both cosmic speed up and missing matter represent signals of a breakdown in our understanding of gravitation law so that one should consider the possibility that the Hilbert -Einstein Lagrangian, linear in the Ricci scalar $R$, should be generalized. Following this line of thinking, the choice of a generic function $f(R)$ can be derived by matching the data and by the "economic" requirement that no exotic ingredients have to be added. This is the underlying philosophy of what is referred to as $f(R)$-gravity (see [7] and references therein). In this context, the same cosmological constant could be removed as an ingredient of the cosmic pie being nothing else but a particular eigenvalue of a general class of theories [12].

However $f(R)$-gravity can be encompassed in the Extended Theories of Gravity being a "minimal" extension of GR where (analytical) functions of Ricci scalar are taken into account. Although higher order gravity theories have received much attention in cosmology, since they are naturally able to give rise to accelerating expansions (both in the late and in the early Universe) and systematic studies of the phase space of solutions are in progress [13], it is possible to demonstrate that $f(R)$-theories can also play a major role at astrophysical scales. In fact, modifying the gravity Lagrangian can affect the gravitational potential in the low energy limit. Provided that the modified potential reduces to the Newtonian one at Solar System scales, this implication could represent an intriguing opportunity rather than a shortcoming for $f(R)$-theories [14]. A corrected gravitational potential could offer the possibility to fit the galaxy rotation curves and the density profiles of galaxy clusters without the need of dark matter $[15,16]$.

In this paper, we want to give an overview of the main features of $f(R)$-gravity theories considering also torsion. After reviewing the basic definitions of torsion and outlying the possibility of a classification in Sec.2, we discuss the field equations of $f(R)$-gravity with torsion in vacuo (Sec.3) and in presence of perfect fluid matter (Sec.4). Analogies and differences with respect to the Palatini approach in $\mathbf{V}_{4}$ are stressed. The equivalence of such theories with Scalar-Tensor Gravity is discussed in Sec.5, while the Cauchy problem for $f(R)$-gravity in presence of perfect-fluid matter is discussed in Sec.6. Conclusions are drawn in Sec.7.

\section{Generalities on torsion}

In this section, we give general features of torsion and associated quantities that are defined in $\mathbf{U}_{4}$ spacetimes [1]. After, we will apply this formalism in the framework of $f(R)$-gravity.

Torsion tensor $S_{a b}{ }^{c}$ is the antisymmetric part of the affine connection coefficients $\Gamma_{a b}^{c}$, that is

$$
S_{a b}^{c}=\frac{1}{2}\left(\Gamma_{a b}^{c}-\Gamma_{b a}^{c}\right) \equiv \Gamma_{[a b]}^{c},
$$

where $a, b, c=0, \ldots 3$. In GR, it is postulated that $S_{a b}{ }^{c}=0$. It is a general convention to call $\mathbf{U}_{4}$ a 4 dimensional space-time manifold endowed with metric and torsion. The manifolds with metric and without 
torsion are labelled as $\mathbf{V}_{4}$. In general, torsion occurs in linear combinations as the contortion tensor

$$
K_{a b}{ }^{c}=-S_{a b}{ }^{c}-S_{a b}^{c}+S_{b}{ }^{c} a=-K_{a b}{ }^{c},
$$

and the modified torsion tensor

$$
T_{a b}{ }^{c}=S_{a b}{ }^{c}+2 \delta_{[a}{ }^{c} S_{b]}
$$

where $S_{a} \equiv S_{a b}{ }^{b}$. According to these definitions, the affine connection can be written as

$$
\Gamma_{a b}^{c}=\left\{\begin{array}{l}
c \\
a b
\end{array}\right\}-K_{a b}{ }^{c},
$$

where $\left\{\begin{array}{l}c \\ a b\end{array}\right\}$ are the usual Christoffel symbols of the symmetric connection. The presence of torsion in the affine connection implies that the covariant derivatives of a scalar field $\phi$ do not commute, that is

$$
\tilde{\nabla}_{[a} \tilde{\nabla}_{b]} \phi=-S_{a b}{ }^{c} \tilde{\nabla}_{c} \phi
$$

and for a vector $v^{a}$ and a covector $w_{a}$, the following relations

$$
\left(\tilde{\nabla}_{a} \tilde{\nabla}_{b}-\tilde{\nabla}_{b} \tilde{\nabla}_{a}\right) v^{c}=R_{a b d}{ }^{c} v^{d}-2 S_{a b}{ }^{d} \tilde{\nabla}_{d} v^{c},
$$

and

$$
\left(\tilde{\nabla}_{a} \tilde{\nabla}_{b}-\tilde{\nabla}_{b} \tilde{\nabla}_{a}\right) w_{d}=R_{a b c}{ }^{d} w_{d}-2 S_{a b}{ }^{d} \tilde{\nabla}_{d} w_{c}
$$

hold. Here $R_{a b c}{ }^{d}$ is the Riemann tensor. The contribution to the Riemann tensor of torsion can be explicitly given by

$$
R_{a b c}{ }^{d}=R_{a b c}{ }^{d}(\{\})-\nabla_{a} K_{b c}{ }^{d}+\nabla_{b} K_{a c}{ }^{d}+K_{a e}{ }^{d} K_{b c}{ }^{e}-K_{b e}{ }^{d} K_{a c}{ }^{e}
$$

where $R_{a b c}{ }^{d}(\{\})$ is the tensor generated by the Christoffel symbols. The symbols $\tilde{\nabla}$ and $\nabla$ have been used to indicate the covariant derivative with and without torsion respectively. From Eq.(2.8), the expressions for the Ricci tensor and the curvature scalar are

$$
R_{a b}=R_{a b}(\{\})-2 \nabla_{a} S_{c}+\nabla_{b} K_{a c}{ }^{b}+K_{a e}{ }^{b} K_{b c}{ }^{e}-2 S_{e} K_{a c}{ }^{e}
$$

and

$$
R=R(\{\})-4 \nabla_{a} S^{a}+K_{c e b} K^{b c e}-4 S_{a} S^{a} .
$$

Torsion can be decomposed with respect to the Lorentz group into three irreducible tensors

$$
S_{a b}{ }^{c}={ }^{T} S_{a b}{ }^{c}+{ }^{A} S_{a b}{ }^{c}+{ }^{V} S_{a b}{ }^{c} .
$$

Torsion has 24 components, of which ${ }^{T} S_{a b}$ has 16 components, ${ }^{A} S_{a b}$ has 4 and ${ }^{V} S_{a b}$ has the remaining 4 components. One has

$$
{ }^{V} S_{a b}{ }^{c}=\frac{1}{3}\left(S_{a} \delta_{b}^{c}-S_{b} \delta_{a}^{c}\right),
$$

where $S_{a}=S_{a b}{ }^{b}$,

$$
{ }^{A} S_{a b}{ }^{c}=g^{c d} S_{[a b d]}
$$

that is called the axial (or totally anti-symmetric) torsion, and

$$
{ }^{T} S_{a b}{ }^{c}=S_{a b}{ }^{c}-{ }^{A} S_{a b}{ }^{c}-{ }^{V} S_{a b}{ }^{c}
$$

that is the traceless non-totally anti-symmetric part of torsion. It is immediately clear that relating torsion to the spin-density of matter is only one of the possible aspects of the problem [8]. 


\section{Field equations in vacuo}

In the metric-affine formulation of $f(R)$-gravity, the dynamical fields are pairs $(g, \Gamma)$ where $g$ is the metric and $\Gamma$ is the linear connection. Considering torsion means to assume a general non-symmetric connection. In vacuo, the field equations are obtained by varying with respect to the metric and the connection the following action

$$
\mathcal{A}(g, \Gamma)=\int \sqrt{|g|} f(R) d s
$$

where $f(R)$ is a real function, $R(g, \Gamma)=g^{i j} R_{i j}$ (with $R_{i j}:=R_{i h j}^{h}$ ) is the scalar curvature associated to the dynamical connection $\Gamma$. More precisely, in the approach with torsion, one can ask for a metric connection $\Gamma$ with torsion different from zero while, in the Palatini approach, $\Gamma$ is non-metric but torsion is null. In vacuo, the field equations for $f(R)$-gravity with torsion are [17-19]

$$
\begin{aligned}
& f^{\prime}(R) R_{i j}-\frac{1}{2} f(R) g_{i j}=0, \\
& T_{i j}{ }^{h}=-\frac{1}{2 f^{\prime}} \frac{\partial f^{\prime}}{\partial x^{p}}\left(\delta_{i}^{p} \delta_{j}^{h}-\delta_{j}^{p} \delta_{i}^{h}\right),
\end{aligned}
$$

while the field equations for $f(R)$-gravity à la Palatini are

$$
\begin{aligned}
& f^{\prime}(R) R_{i j}-\frac{1}{2} f(R) g_{i j}=0 \\
& \nabla_{k}\left(f^{\prime}(R) g_{i j}\right)=0
\end{aligned}
$$

In both cases, considering the trace of Einstein-like field equations (3.16a) and (3.17a), one gets the relation

$$
f^{\prime}(R) R-2 f(R)=0
$$

The latter is an identity automatically satisfied by all possible values of $R$ only in the special case $f(R)=$ $\alpha R^{2}$. In all other cases, equation (3.18) represents a constraint on the scalar curvature $R$. Therefore, if $f(R) \neq \alpha R^{2}$, the scalar curvature $R$ is a constant coinciding with the solution of Eq. 3.18). In this case, Eqs. 3.16b and 3.17b imply that both dynamical connections coincide with the Levi-Civita connection associated to the metric $g_{i j}$ that is solution of the Einstein-like field equations. In other words, both theories reduce to the Einstein theory with cosmological constant. On the contrary, only in the case $f(R)=\alpha R^{2}$ metric-affine $f(R)$-theories differ from GR in vacuo. To see this point, let us consider the theory with torsion: from Eqs. 3.16), we obtain final field equations of the form

$$
\tilde{R}_{i j}-\frac{1}{4} \tilde{R} g_{i j}=-\frac{2}{3} \tilde{\nabla}_{j} T_{i}+\frac{1}{6} \tilde{\nabla}_{h} T^{h} g_{i j}-\frac{2}{9} T_{i} T_{j}+\frac{1}{18} T_{h} T^{h} g_{i j}
$$

and

$$
\frac{\partial}{\partial x^{i}}\left(\tilde{R}+2 \tilde{\nabla}_{h} T^{h}-\frac{2}{3} T_{h} T^{h}\right)=-\frac{2}{3}\left(\tilde{R}+2 \tilde{\nabla}_{h} T^{h}-\frac{2}{3} T_{h} T^{h}\right) T_{i}
$$

where $\tilde{R}_{i j}$ and $\tilde{R}$ are respectively the Ricci tensor and scalar associated with the metric $g_{i j}, \tilde{\nabla}_{h}$ denotes the Levi-Civita covariant derivative induced by $g_{i j}$ and $T_{i}:=T_{i j}{ }^{j}$ is the trace of the above defined modified torsion tensor. 


\section{Field equations in presence of matter}

In presence of matter, the field equations are derived from an action functional of the form

$$
\mathcal{A}(g, \Gamma)=\int\left(\sqrt{|g|} f(R)+\mathcal{L}_{m}\right) d s
$$

where $\mathcal{L}_{m}$

/ is a suitable material Lagrangian density. Assuming that the material Lagrangian does not depend on the dynamical connection, the field equations are

$$
\begin{aligned}
& f^{\prime}(R) R_{i j}-\frac{1}{2} f(R) g_{i j}=\Sigma_{i j}, \\
& T_{i j}{ }^{h}=-\frac{1}{2 f^{\prime}(R)} \frac{\partial f^{\prime}(R)}{\partial x^{p}}\left(\delta_{i}^{p} \delta_{j}^{h}-\delta_{j}^{p} \delta_{i}^{h}\right)
\end{aligned}
$$

for $f(R)$-gravity with torsion, and

$$
\begin{aligned}
& f^{\prime}(R) R_{i j}-\frac{1}{2} f(R) g_{i j}=\Sigma_{i j}, \\
& \nabla_{k}\left(f^{\prime}(R) g_{i j}\right)=0
\end{aligned}
$$

for $f(R)$-gravity in the Palatini approach. In Eqs. 4.22a and 44.23a , the quantity $\Sigma_{i j}:=-\frac{1}{\sqrt{|g|}} \frac{\delta \mathcal{L}_{m}}{\delta g^{i j}}$ is the stress-energy tensor. Considering the trace of Eqs. 4.22a and 4.23a), we obtain a relation between the curvature scalar $R$ and the trace of the stress-energy tensor $\Sigma:=g^{i j} \Sigma_{i j}$. We have indeed

$$
f^{\prime}(R) R-2 f(R)=\Sigma .
$$

From now on, we shall suppose that the relation (4.24) is invertible as well as that $\Sigma \neq$ const. Under these hypotheses, the curvature scalar $R$ can be expressed as a suitable function of $\Sigma$, namely

$$
R=F(\Sigma) \text {. }
$$

Starting from this result and defining the scalar field

$$
\varphi:=f^{\prime}(F(\Sigma))
$$

we can put the field equations of both Palatini and with torsion theories in the same form [17,20], that is

$$
\begin{array}{r}
\tilde{R}_{i j}-\frac{1}{2} \tilde{R} g_{i j}=\frac{1}{\varphi} \Sigma_{i j}+\frac{1}{\varphi^{2}}\left(-\frac{3}{2} \frac{\partial \varphi}{\partial x^{i}} \frac{\partial \varphi}{\partial x^{j}}+\varphi \tilde{\nabla} j \frac{\partial \varphi}{\partial x^{i}}+\frac{3}{4} \frac{\partial \varphi}{\partial x^{h}} \frac{\partial \varphi}{\partial x^{k}} g^{h k} g_{i j}\right. \\
\left.-\varphi \tilde{\nabla}^{h} \frac{\partial \varphi}{\partial x^{h}} g_{i j}-V(\varphi) g_{i j}\right),
\end{array}
$$

where we have introduced the effective potential

$$
V(\varphi):=\frac{1}{4}\left[\varphi F^{-1}\left(\left(f^{\prime}\right)^{-1}(\varphi)\right)+\varphi^{2}\left(f^{\prime}\right)^{-1}(\varphi)\right]
$$

for the scalar field $\varphi$. In Eq. 44.27, $\tilde{R}_{i j}, \tilde{R}$ and $\tilde{\nabla}$ denote respectively the Ricci tensor, the scalar curvature and the covariant derivative associated with the Levi-Civita connection of the dynamical metric 
$g_{i j}$. Therefore, if the dynamical connection $\Gamma$ is not coupled with matter, both theories (with torsion and Palatini-like) generate identical Einstein-like field equations. On the contrary, the field equations for the dynamical connection are different and (in general) give rise to different solutions. In fact, the connection $\Gamma$ solution of Eqs. (4.22b) is

$$
\Gamma_{i j}{ }^{h}=\tilde{\Gamma}_{i j}{ }^{h}+\frac{1}{2 \varphi} \frac{\partial \varphi}{\partial x^{j}} \delta_{i}^{h}-\frac{1}{2 \varphi} \frac{\partial \varphi}{\partial x^{p}} g^{p h} g_{i j}
$$

where $\tilde{\Gamma}_{i j}^{h}$ denote the coefficients of the Levi-Civita connection associated with the metric $g_{i j}$, while the connection $\bar{\Gamma}$ solution of Eqs. 4.23b is

$$
\bar{\Gamma}_{i j}^{h}=\tilde{\Gamma}_{i j}^{h}+\frac{1}{2 \varphi} \frac{\partial \varphi}{\partial x^{j}} \delta_{i}^{h}-\frac{1}{2 \varphi} \frac{\partial \varphi}{\partial x^{p}} g^{p h} g_{i j}+\frac{1}{2 \varphi} \frac{\partial \varphi}{\partial x^{i}} \delta_{j}^{h},
$$

and coincides with the Levi-Civita connection induced by the conformal metric $\bar{g}_{i j}:=\varphi g_{i j}$. By comparison, the connections $\Gamma$ and $\bar{\Gamma}$ satisfy the relation

$$
\bar{\Gamma}_{i j}^{h}=\Gamma_{i j}{ }^{h}+\frac{1}{2 \varphi} \frac{\partial \varphi}{\partial x^{i}} \delta_{j}^{h} .
$$

Of course, the Einstein-like equations (4.27) are coupled with the matter field equations. In this respect, it is worth pointing out that Eqs. (4.27) imply the same conservation laws holding in GR. We have in fact [21,22]

Proposition 4.1 Eqs. (4.26), 4.27) and 4.28) imply the standard conservation laws $\tilde{\nabla}^{j} \Sigma_{i j}=0$.

Summarizing all the results, we can say that if both the theories (with torsion and Palatini-like) are considered as "metric", in the sense that the dynamical connection $\Gamma$ is not coupled with matter $\left(\frac{\delta \mathcal{L}_{m}}{\delta \Gamma}=0\right)$ and it does not define parallel transport and covariant derivative in space-time, then the two approaches are completely equivalent. Indeed, in the "metric" framework, the true connection of space-time is the Levi-Civita one associated with the metric $g$ and the role played by the dynamical connection $\Gamma$ is just to generate the right Einstein-like equations of the theory. On the contrary, if the theories are genuinely metric-affine, then they are different even though the condition $\frac{\delta \mathcal{L}_{m}}{\delta \Gamma}=0$ is satisfied.

At this point, some considerations are due in relation to conformal transformations. If the trace $\Sigma$ of the stress-energy tensor is independent of the metric $g_{i j}$, Eqs. 4.27) can be simplified by passing from the Jordan to the Einstein frame. Indeed, performing the conformal transformation $\bar{g}_{i j}=\varphi g_{i j}$, it is easily seen that Eqs. 4.27) assume the simpler form (see also [17,20])

$$
\bar{R}_{i j}-\frac{1}{2} \bar{R} \bar{g}_{i j}=\frac{1}{\varphi} \Sigma_{i j}-\frac{1}{\varphi^{3}} V(\varphi) \bar{g}_{i j},
$$

where $\bar{R}_{i j}$ and $\bar{R}$ are respectively the Ricci tensor and the curvature scalar derived from the conformal metric $\bar{g}_{i j}$. As mentioned above, the conformal transformation is working if the trace of the stress-energy tensor is independent of the metric $g_{i j}$. Only in this case, Eqs. 4.32) depend exclusively on the conformal metric $\bar{g}_{i j}$ and the other matter fields. The quantity

$$
T_{i j}:=\frac{1}{\varphi} \Sigma_{i j}-\frac{1}{\varphi^{3}} V(\varphi) \bar{g}_{i j}
$$

appearing in the right-hand side of Eqs. (4.32), plays the role of effective stress-energy tensor for the conformally transformed theory. The existing relation between the conservation laws, holding in the Jordan and in the Einstein frame separately, is explained by the following [21,22]

Proposition 4.2 Given the Levi-Civita connection $\bar{\Gamma}$ derived from the conformal metric tensor $\bar{g}$ and given the associated covariant derivative $\bar{\nabla}$, the condition $\bar{\nabla}^{j} T_{i j}=0$ is equivalent to the condition $\tilde{\nabla}^{j} \Sigma_{i j}=0$.

This result will be particularly useful for the well-formulation and the well-position of the Cauchy problem. 


\section{Equivalence with scalar-tensor theories}

The action functional of a (purely metric) scalar-tensor theory is given by

$$
\mathcal{A}(g, \varphi)=\int\left[\sqrt{|g|}\left(\varphi \tilde{R}-\frac{\omega_{0}}{\varphi} \varphi_{i} \varphi^{i}-U(\varphi)\right)+\mathcal{L}_{m}\right] d s
$$

where $\varphi$ is the scalar field, $\varphi_{i}:=\frac{\partial \varphi}{\partial x^{i}}$ and $U(\varphi)$ is the potential of $\varphi$. The matter Lagrangian $\mathcal{L}_{m}\left(g_{i j}, \psi\right)$ is a function of the metric and some matter fields $\psi \cdot \omega_{0}$ is the so called Brans-Dicke parameter. The field equations, derived by varying with respect to the metric and the scalar field, are

$$
\tilde{R}_{i j}-\frac{1}{2} \tilde{R} g_{i j}=\frac{1}{\varphi} \Sigma_{i j}+\frac{\omega_{0}}{\varphi^{2}}\left(\varphi_{i} \varphi_{j}-\frac{1}{2} \varphi_{h} \varphi^{h} g_{i j}\right)+\frac{1}{\varphi}\left(\tilde{\nabla}_{j} \varphi_{i}-\tilde{\nabla}_{h} \varphi^{h} g_{i j}\right)-\frac{U}{2 \varphi} g_{i j}
$$

and

$$
\frac{2 \omega_{0}}{\varphi} \tilde{\nabla}_{h} \varphi^{h}+\tilde{R}-\frac{\omega_{0}}{\varphi^{2}} \varphi_{h} \varphi^{h}-U^{\prime}=0
$$

where $\Sigma_{i j}:=-\frac{1}{\sqrt{|g|}} \frac{\delta \mathcal{L}_{m}}{\delta g^{i j}}$ and $U^{\prime}:=\frac{d U}{d \varphi}$. Taking the trace of Eq. (5.35) and using it to replace $\tilde{R}$ in Eq. (5.36), one obtains the equation

$$
\left(2 \omega_{0}+3\right) \tilde{\nabla}_{h} \varphi^{h}=\Sigma+\varphi U^{\prime}-2 U .
$$

By a direct comparison, it is immediately seen that for

$$
\omega_{0}=-\frac{3}{2} \quad \text { and } \quad U(\varphi)=\frac{2}{\varphi} V(\varphi)
$$

Eqs. 5.35) become formally identical to the Einstein-like equations 4.27) for a metric-affine $f(R)$ theory. Moreover, in such a circumstance, Eq. (5.37) reduces to the algebraic equation

$$
\Sigma+\varphi U^{\prime}-2 U=0
$$

relating the matter trace $\Sigma$ to the scalar field $\varphi$, just as it happens for $f(R)$ theories. More in particular, it is a straightforward matter to verify that (under the condition $f^{\prime \prime} \neq 0$ [17]) Eq. (5.39) expresses exactly the inverse relation of

$$
\varphi=f^{\prime}(F(\Sigma)) \text { namely } \Sigma=F^{-1}\left(\left(f^{\prime}\right)^{-1}(\varphi)\right),
$$

being $F^{-1}(X)=f^{\prime}(X) X-2 f(X)$. In conclusion, it follows that, in the "metric" interpretation, metricaffine $f(R)$ - theories (with torsion or Palatini) are equivalent to scalar-tensor theories with Brans-Dicke parameter $\omega_{0}=-\frac{3}{2}$. It is also possible to show the equivalence between $f(R)$ theories and $\omega_{0}=0$ scalar-tensor theories with torsion. To this end, let us consider an action functional of the kind

$$
\mathcal{A}(g, \Gamma, \varphi)=\int\left[\sqrt{|g|}(\varphi R-U(\varphi))+\mathcal{L}_{m}\right] d s
$$

where the dynamical fields are respectively a metric $g_{i j}$, a metric connection $\Gamma_{i j}{ }^{k}$ and a scalar field $\varphi$. Action 5.41 describes a scalar-tensor theory with torsion and parameter $\omega_{0}=0$. The variation with respect to $\varphi$ yields the first field equation

$$
R=U^{\prime}(\varphi)
$$

while variations with respect to the metric and the connection give rise to the resulting field equations

$$
T_{i j}{ }^{h}=-\frac{1}{2 \varphi} \frac{\partial \varphi}{\partial x^{p}}\left(\delta_{i}^{p} \delta_{j}^{h}-\delta_{j}^{p} \delta_{i}^{h}\right)
$$


and

$$
R_{i j}-\frac{1}{2} R g_{i j}=\frac{1}{\varphi} \Sigma_{i j}-\frac{1}{2 \varphi} U(\varphi) g_{i j} .
$$

Inserting the content of Eq. (5.42) in the trace of Eq. (5.44), we obtain again the algebraic ralation between $\Sigma$ and $\varphi$, that is

$$
\Sigma-2 U(\varphi)+\varphi U^{\prime}(\varphi)=0
$$

identical to Eq. (5.39). As above, choosing the potential $U(\varphi)=\frac{2}{\varphi} V(\varphi)$, it is easily seen that Eq. (5.45) is equivalent to the relation $\varphi=f^{\prime}(F(\Sigma))$ defining the scalar field $\varphi$ in terms of the trace $\Sigma$. In view of this, decomposing $R_{i j}$ and $R$ in their Christoffel and torsion dependent terms, Eqs. (5.43) and (5.44) become identical to the field equations 4.22 . As mentioned previously, this fact shows the equivalence between $f(R)$ theories and $\omega_{0}=0$ scalar-tensor theories with torsion, in the metric-affine framework.

\section{The Cauchy problem in presence of perfect fluid matter}

Let us consider now metric-affine $f(R)$ theories coupled with a perfect fluid. We shall show that, in the Einstein frame, the analysis of the Cauchy problem can be carried out following the same arguments developed in [23]. The result is that metric-affine $f(R)$ theories, coupled with perfect fluid matter, possess a well formulated and well posed Cauchy problem depending on the form of $f(R)$.

To see this point, we start by looking for a metric $g_{i j}$ of signature $(-+++)$ in the Jordan frame, solution of Eqs. 4.27). The stress-energy tensor will be of the form

$$
\Sigma_{i j}=(\rho+p) U_{i} U_{j}+p g_{i j},
$$

with corresponding matter field equations given by

$$
\tilde{\nabla}_{j} \Sigma^{i j}=0 .
$$

In Eqs. (6.46), the scalars $\rho$ and $p$ denote respectively the matter-energy density and the pressure of the fluid, while $U_{i}$ indicate the four velocity of the fluid, satisfying the obvious condition $g^{i j} U_{i} U_{j}=-1$. After performing the conformal transformation $\bar{g}_{i j}=\varphi g_{i j}$ (which is working since the trace $\Sigma$ is independet of the metric $g_{i j}$ ), we can express the field equations in the Einstein frame as

$$
\bar{R}_{i j}-\frac{1}{2} \bar{R} \bar{g}_{i j}=T_{i j},
$$

and

$$
\bar{\nabla}_{j} T^{i j}=0
$$

where

$$
T_{i j}=\frac{1}{\varphi}(\rho+p) U_{i} U_{j}+\left(\frac{p}{\varphi^{2}}-\frac{V(\varphi)}{\varphi^{3}}\right) \bar{g}_{i j},
$$

is the effective stress-energy tensor. In view of Proposition 4.2, Eqs. 6.47b) are equivalent to Eqs. (6.46b). This is a key point in our discussion, allowing us to apply, to the present case, the results achieved in [23]. Moreover, for simplicity, we shall suppose that the scalar field $\varphi$ is positive, that is $\varphi>0$. The opposite case $\varphi<0$, differing from the former only for some technical aspects, will be briefly discussed after. Under the above assumption, the four velocity of the fluid in the Einstein frame can be expressed as $\bar{U}_{i}=\sqrt{\varphi} U_{i}$. In view of this, the stress-energy tensor $(6.48)$ can be rewritten, in terms of the four velocity $\bar{U}_{i}$, as

$$
T_{i j}=\frac{1}{\varphi^{2}}(\rho+p) \bar{U}_{i} \bar{U}_{j}+\left(\frac{p}{\varphi^{2}}-\frac{V(\varphi)}{\varphi^{3}}\right) \bar{g}_{i j} .
$$


Furthermore, introducing the effective mass-energy density

$$
\bar{\rho}:=\frac{\rho}{\varphi^{2}}+\frac{V(\varphi)}{\varphi^{3}}
$$

and the effective pressure

$$
\bar{p}:=\frac{p}{\varphi^{2}}-\frac{V(\varphi)}{\varphi^{3}}
$$

the stress-energy tensor 6.49 assumes the final standard form

$$
T_{i j}=(\bar{\rho}+\bar{p}) \bar{U}_{i} \bar{U}_{j}+\bar{p} \bar{g}_{i j} .
$$

It is worth noticing that, starting from an equation of state of the form $\rho=\rho(p)$ and assuming that the relation 6.50b is invertible $(p=p(\bar{p}))$, by composition with Eq. 6.50a , we derive an effective equation of state $\bar{\rho}=\bar{\rho}(\bar{p})$. In addition to this, we recall that the explicit expression of the scalar field $\varphi$ as well as of the potential $V(\varphi)$ are directly related with the particular form of the function $f(R)$. Then, the requirement of invertibility of the relation $6.50 \mathrm{~b}$ together with the condition $\varphi>0$ (or, equivalently, $\varphi<0$ ) become criteria for the viability of the functions $f(R)$. In other words, they provide us with precise rules of selection for the admissible functions $f(R)$. From now on, the treatment of the Cauchy problem can proceed step by step as in [23]. We only recall the conclusion stated in [23], where it is proved that the Cauchy problem for the system of differential equations 6.47, with stress-energy tensor given by Eq. 6.51) and equation of state $\bar{\rho}=\bar{\rho}(\bar{p})$, is well-posed if the condition

$$
\frac{d \bar{\rho}}{d \bar{p}} \geq 1
$$

is satisfied. We stress that, in order to satisfy the condition 6.52, we do not need to invert explicitly the relation 6.50b), but more simply, we have to verify

$$
\frac{d \bar{\rho}}{d \bar{p}}=\frac{d \bar{\rho} / d p}{d \bar{p} / d p} \geq 1
$$

directly from the expressions 6.50) and the equation of state $\rho=\rho(p)$. Once again, the condition 6.53), depending on the peculiar expressions of $\varphi$ and $V(\varphi)$, is strictly related to the particular form of the function $f(R)$. Then, condition 6.53) represents a further criterion for the viability of the functions $f(R)$. For the sake of completeness, we outline the case $\varphi<0$. We still suppose that the signature of the metric in the Jordan frame is $(-+++)$. Therefore, the signature of the conformal metric will be $(+---)$ and the four velocity of the fluid in the Einstein frame will be $\bar{U}_{i}=\sqrt{-\varphi} U_{i}$. The effective stress-energy tensor will be given now by

$$
T_{i j}=-\frac{1}{\varphi^{2}}(\rho+p) \bar{U}_{i} \bar{U}_{j}+\left(\frac{p}{\varphi^{2}}-\frac{V(\varphi)}{\varphi^{3}}\right) \bar{g}_{i j}=(\bar{\rho}+\bar{p}) \bar{U}_{i} \bar{U}_{j}-\bar{p} \bar{g}_{i j}
$$

where we have introduced the quantities

$$
\bar{\rho}:=-\frac{\rho}{\varphi^{2}}-\frac{V(\varphi)}{\varphi^{3}}
$$

and

$$
\bar{p}:=-\frac{p}{\varphi^{2}}+\frac{V(\varphi)}{\varphi^{3}}
$$

representing, as above, the effective mass-energy density and pressure. At this point, demonstration proceeds again as in [23], except for a technical aspect. The fact quantity $r:=\bar{\rho}+\bar{p}=-\frac{\rho+p}{\varphi^{2}}$ this time is negative (if, as usual, $\rho$ and $p$ are assumed positive). Therefore, instead of using the function $\log \left(f^{-2} r\right)$ as in [23], we need to use $\log \left(-f^{-2} r\right)$. The reader can easily verify that, with this choice, the arguments in [23] apply equally well. 


\section{Discussion and Conclusions}

$f(R)$-gravity seems a viable approach to solve some shortcomings coming from GR, in particular problems related to quantization on curved spacetime and cosmological issues related to early Universe (inflation) and late time dark components. It is worth noticing that the scheme of GR is fully preserved and $f(R)$ can be considered a straightforward extension where the gravitational action has not to be necessarily linear in the Ricci scalar $R$. We have discussed the possibility that also the torsion field could play an important role in the dynamics being the $\mathbf{U}_{4}$ manifolds a generalization of the pseudo-Riemannian manifolds $\mathbf{V}_{4}$ (torsionless) usually adopted in GR. Torsion field, in the metric-affine formalism, plays a fundamental role in clarifying the relations between the Palatini and the metric approaches: it gives further degrees of freedom which contribute, together with curvature degrees of freedom, to the dynamics. The aim is to achieve a self-consistent theory where unknown ingredients as dark energy and dark matter (up to now not detected at a fundamental level) could be completely "geometrized". Torsion field assumes a relevant role in presence of standard matter since it allows to establish a definite equivalence between scalar-tensor theories and $f(R)$-gravity, also in relation to conformal transformations. In this case, the Chauchy problem results well formulated and well posed depending on the form of $f(R)$ [22]. Furthermore, from a cosmological viewpoint, torsion field could dynamically trigger the amount of dark components giving a straightforward explanation of the coincidence problem [17].

Acknowledgements We acknowledge our friends R. Cianci and C. Stornaiolo for useful comments and results achieved together.

\section{References}

[1] F.W. Hehl, P. von der Heyde, G.D. Kerlick and J.M. Nester, Rev. Mod. Phys. 48 (1976) 393.

[2] M.B. Green, J.H. Schwarz, E. Witten, Superstring Theory (Cambridge University Press, Cambridge, 1987).

[3] Yu A. Kubyshin, J. Math. Phys. 35 (1994) 310.

[4] P.S. Howe, G. Papadopoulos, Phys. Lett. 379B (1996) 80.

[5] G. Papadopoulos, P.K. Townsend, Nucl. Phys. B444 (1995) 245.

[6] V. De Sabbata, C. Sivaram, Astr. and Space Sci. 176 (1991) 141.

[7] S. Nojiri, S. D. Odintsov Int.J.Geom.Meth.Mod.Phys. 4 (2007) 115.

S. Capozziello, M. Francaviglia, Gen. Rel. Grav. 40: (2008) 357.

T. P. Sotiriou, V. Faraoni, arXiv:0805.1726 [gr-qc] (2008).

[8] S. Capozziello, G. Lambiase and C. Stornaiolo, Annals. Phys. (Leipzig) 10 (2001) 713.

[9] J. Barrow and A.C. Ottewill, J. Phys. A: Math. Gen. 16 (1983) 2757.

[10] A.A. Starobinsky, Phys. Lett. 91B (1980) 99.

[11] C.M. Will, Theory and Experiments in Gravitational Physics (1993) Cambridge Univ. Press, Cambridge.

[12] S. Capozziello, R. Garattini, Class. Quant. Grav. 24 (2007) 1627.

[13] S. Capozziello, S. Nojiri, S.D. Odintsov, A. Troisi. Phys. Lett. 639 B (2006) 135.

[14] S. Capozziello, E. Piedipalumbo, C. Rubano, P. Scudellaro. Astron. \& Astroph. 505 (2009) 21.

[15] S. Capozziello, V.F. Cardone, A. Troisi, MNRAS 375 (2007) 1423.

[16] S. Capozziello, E. De Filippis, V. Salzano, MNRAS 394 (2009) 947.

[17] S. Capozziello, R. Cianci, C. Stornaiolo and S. Vignolo, Class. Quantum Grav., 24 (2007) 6417.

[18] S. Capozziello, R. Cianci, C. Stornaiolo and S. Vignolo, Int. J. Geom. Methods Mod. Phys. 5 (2008) 765.

[19] S. Capozziello, R. Cianci, C. Stornaiolo and S. Vignolo, Physica Scripta 78 (2008) 065010.

[20] G. J. Olmo, Phys. Rev. D 72 (2005) 083505.

[21] S. Capozziello and S. Vignolo, Int. J. Geom. Methods Mod. Phys. 6 (2009) 985.

[22] S. Capozziello and S. Vignolo, Class. Quantum. Grav. 26 (2009) 175013.

[23] Y. Fourés-Bruhat, Bull. de la S.M.F., 86 (1958) 155. 